\title{
Determinación de zonas inundables con HEC-RAS en zonas de la Cordillera Central de los Andes. Microcuenca del río Saraus- río La Llanga. Celendín
}

\author{
Determination of flood zones with HEC-RAS in zones of the Central Andes \\ mountain range. Microcuenca Saraus-La Llanga river. Celendin
}

\author{
Alejandro Alcántara Boza', Any Castro Tenorio² \\ Recibido: 10/03/2021 - Aprobado: 16/06/2021 - Publicado: 23/12/2021
}

\begin{abstract}
RESUMEN
Las inundaciones son un fenómeno estacional que ocurre en espacios con tormentas ocasionales y influenciadas por características topográficas del terreno. Existen pocos estudios de zonas de inundación en la Cordillera de los Andes, por ello en el presente estudio se utilizó modelos de simulación hidráulica para identificar zonas de riesgo y desastres. Hec-Ras es un modelo hidrológico de inundaciones usado para para flujos inestables, y bajo los periodos de retorno de 30, 50 y 100 años para flujo estables, en la microcuenca del río Saraus. El modelo utiliza ecuaciones diferenciales del tipo determinístico que nos permite pronosticar la dinámica de los niveles de agua por eventos hidrometeorológicos extremos. Se empleó diversas herramientas geoespaciales como el HEC-RAS, RasMapper, datos geométricos de TIN obtenido de un modelo de elevación digital (DEM/SRTM), imágenes satelitales y Sistemas de Información Geográfica. Para la simulación hidráulica se trazaron 26 secciones transversales separadas a 300 metros. Las manchas simuladas de inundaciones anuales del río brindan información sobre el efecto que generan las inundaciones para tres periodos de retorno de 30, 50 y 100 años. Las zonas de riesgo con mayor impacto por inundaciones corresponden a los inicios de la cuenca, alcanzando cotas de hasta $10 \mathrm{~m}$ y anchos de hasta $140 \mathrm{~m}$; algunas áreas están destinadas a actividades agrícolas, lo que genera pérdidas de cultivos principalmente y pérdidas económicas e incluso vidas humanas lo cual en muchos casos pasa por desapercibido por falta de información y gestión en cuencas hidrográficas de altamontaña.
\end{abstract}

Palabras claves: Hec-Ras; microcuenca Saraus; inundación; cordillera andina; río La Llanga.

\begin{abstract}
Floods are a seasonal phenomenon that occurs in spaces with occasional storms and influenced by topographic characteristics of the terrain. There are few studies of flood zones in the Andes Mountains, therefore in this study hydraulic simulation models were used to identify risk and disaster areas. Hec-Ras is a hydrological flood model used for unstable flows, and under return periods of 30,50 and 100 years for stable flows, in the microbasin of the Saraus River. The model uses differential equations of the deterministic type that allow us to forecast the dynamics of water levels due to extreme hydrometeorological events. Various geospatial tools were used such as HEC-RAS, RasMapper, geometric TIN data obtained from a digital elevation model (DEM / SRTM), satellite images and Geographic Information Systems. For the hydraulic simulation, 26 cross sections were trace 300 meters apart. The simulated stains of annual river floods provide information on the effect of flooding for three return periods of 30,50 and 100 years. The risk areas with the greatest impact from floods correspond to the beginning of the basin, reaching heights of up to $10 \mathrm{~m}$ with widths of up to 140 $\mathrm{m}$. Some areas are destined for agricultural activities, which generates losses of crops mainly and economic losses and even human lives, which in many cases goes unnoticed due to lack of information and management in high mountain watersheds.
\end{abstract}

Keywords: Hec-Ras; Microcuenca Saraus; flood; Andean Mountain range; La Llanga river.

1 Universidad Nacional Mayor de San Marcos, Facultad de Ingeniería Geológica, Minera, Metalúrgica y Geográfica, Perú, Lima. Docente. Autor para correspondencia: falcantarab@unmsm.edu.pe - ORCID: https://orcid.org/0000-0001-9127-4450

2 Universidad Nacional Mayor de San Marcos, Facultad de Ingeniería Geológica, Minera, Metalúrgica y Geográfica, E.P. Ingeniería Geográfica, Perú, Lima. Estudiante.

E-mail: any.castro@unmsm.edu.pe - ORCID: https://orcid.org/0000-0002-6593-6016 


\section{INTRODUCCIÓN}

La identificación de zonas inundables en ríos primarios de la Cordillera de los Andes es uno de los temas poco conocidos y estudiados y que genera preocupación solo cuando el hecho ha ocurrido, lo que demanda altas pérdidas económicas y sociales. Una forma de conocer y predecir estos fenómenos es mediante los procesos de simulación. Las simulaciones en quebradas y ríos, asume un rol importante de reconocimiento como sistemas de control y amortiguamiento en defensa de procesos naturales que conlleva la geomorfología natural de las estribaciones andinas. Varios son los factores de inundación que se generan en cabeceras de cuenca (Wang et al., 2018); la geomorfología de las estribaciones andinas son factores de lineamiento en la dirección, velocidad y amortiguamiento de flujos de agua por avenidas de agua en épocas de lluvia.

Las inundaciones son un fenómeno que se repite a lo largo de la historia ((Shivaprasad Sharma SV, Parth Sarathi Roy, Chakravarthi V, 2017), debido a procesos inusuales de tormentas que generan descargas de aguas en cabeceras de cuenca y los ríos van recogiendo los flujos de agua de sus colectores, por lo que el fenómeno de inundaciones también es poco predecible en función al cauce y las circunstancias de corrientes de agua que se desplaza en función a la gravedad y al débil obstáculo que ofrece el material acumulado en los cauces de los ríos, llevando consigo sedimentos y escombros (Talha Anees et al., 2019) lo que genera mayor efecto sobre las áreas afectadas.

Poco son atendidos los procesos de control de inundaciones en la serranía de nuestro país, uno de ellos es debido al desconocimiento de las posibles zonas de inundación, la falta de data, monitoreo y la falta de coordinación e integración de sistemas que ayuden a controlar estos procesos (Radzicki et al. (2021); Solín et al. (2017) para lo cual el uso de Sistemas de Información Geográfica, combinado con modelos hidrológicos, son aplicables para determinar índices de peligros de inundación (Rodriguez \& Ortega., 2005; Quisphe Torres, Forge Armando; Sullca Otazu, 2015).

Las inundaciones no son un fenómeno que se debe descuidar en la parte inicial o media de los ríos, si bien no existen grandes poblaciones en estos espacios, pero si generan pérdidas de vidas humanas y sobre todo las pérdidas de áreas cultivadas (Quast et al., 2011) las zonas agrícolas corresponden básicamente a plantaciones permanentes o periódicas; más aún si las condiciones de flujo van arrastrando sedimentos lo que podría generar un enorme desastre ambiental (Fang \& Pomeroy, 2016).

En consecuencia, el objetivo del presente estudio es determinar las zonas de inundación y su recorrido en ríos primarios en la Cordillera de los Andes, por efecto de altas precipitaciones y su proyección en $\mathrm{n}$ años a partir de la data obtenida.

Para ello se ha realizado el uso de sistemas de modelamiento, como parte del proceso de información que necesitan los gestores de recursos de gobiernos regionales y locales. Como parte de los sistemas hidrológicos es necesario el conocimiento de todos los procesos del ciclo hidrológico para llevar a cabo buenos sistemas de simulación, el uso de métodos numéricos y análisis de campo.

\section{MÉTODOS}

El área de estudio se realizó en la parte Central de la Cordillera de los Andes del Norte del Perú. La microcuenca Saraus, pertenece a la cuenca La Llanga, afluente al marañón por la margen izquierda. Geopolíticamente se encuentra en el distrito de La Libertad de Pallán, Provincia de Celendín, región de Cajamarca (Figura 1).
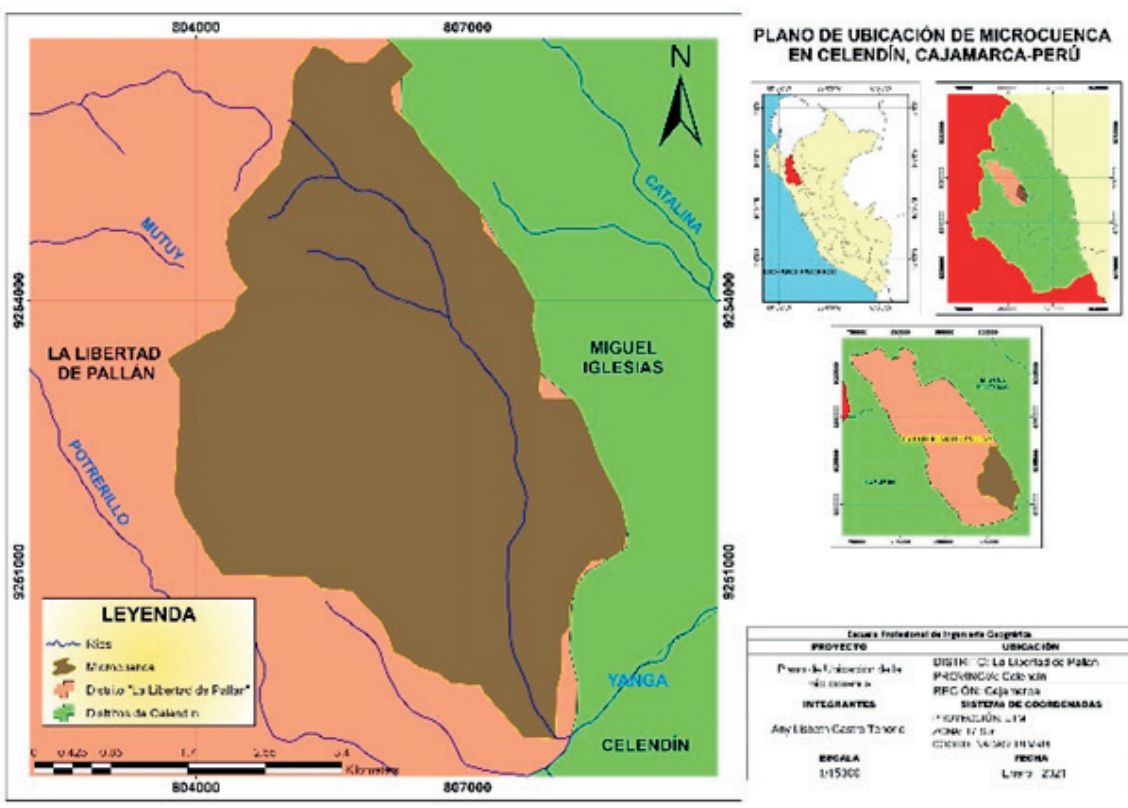

Figura 1. Mapa de Ubicación del Área de Estudio. 
Para los análisis de inundaciones, se utilizó El modelo hidrológico HEC-RAS que es un modelo de base numérica, del Cuerpo de Ingenieros de la Armada de los Estados Unidos, la cual se puede usar de manera libre y tiene continuamente actualizaciones (Turégano, 2012; (Nanía \& Molero, 2007). Este modelo tiene en consideración la ecuación de energía (Ec. 1), considerando pérdidas de energía del flujo de agua por fricción (ecuaciones de Manning); por consiguiente, las pérdidas de energía en tramos es resultado de la contracción y expansiones que sufre el flujo, la cual se calcula como fracción de variación de la velocidad entre dos sectores (Brunner, 2010). Para ello, se determina el tirante en una sección del río (antes o después de un punto de análisis en función a su régimen de escurrimiento). Para el balance de energía, se utiliza la ecuación:

$$
Y_{2+} Z_{2}+\frac{a_{2} v_{2}^{2}}{2 g}=Y_{1+} Z_{1}+\frac{a_{1} v_{1}^{2}}{2 g}+h_{e} \quad \text { Ec. } 1
$$

$\mathrm{Y}_{1}, \mathrm{Y}_{2}=$ tirante de sección trasversal; $\mathrm{Z}_{1}, \mathrm{Z}_{2}=$ altura del fondo en canal principal; $V_{1}, V_{2}=$ velocidades medias; $\mathrm{a}_{1}, \mathrm{a}_{2}=$ coeficientes de velocidad; $\mathrm{g}=$ gravedad, $\mathrm{y}$ he $=$ energía perdída (Ceballos Lopez, 2011).

Según Ardiclioglu and Kuriqi. (2019), en este modelo, el flujo de agua tiene un comportamiento unidimensional, para el tirante y la velocidad se asume que varían en dirección longitudinal del cauce, con un eje aproximadamente en línea recta y una velocidad constante a lo largo de la sección trasversal. Como variables influyentes en inundaciones, se considera: la topografía, caudales máximos anuales y el coeficiente de rugosidad. Para la topografía del espacio de estudio se descargó el DEM del satélite Alos Palsar de la zona de estudio (https://earthdata.nasa.gov/) con resolución espacial de $18 \mathrm{~m}$; se utilizó el software ArcGis 10.2 (Mohamed et al. 2020) y la herramienta Hillshade para mejorar el detalle del relieve del río. Para el estudio se consideró la estación Puente Maygasbamba, de la cual se tomaron datos de caudales máximos anuales (http://snirh. ana.gob.pe/visors2/) del periodo de 1997 al 2014.

Con la herramienta RAS Mapper se digitalizó el cauce del río con una base de imagen satelital exportada de SAS PLANET con el satélite Google. A continuación, se crearon los bancos del cauce del cuerpo de agua (de aguas arriba a aguas de abajo), en el orden de izquierda a derecha considerando la topografía del terreno (Figura 2).

Se crearon 26 secciones transversales, el cual se realizó con la herramienta Cross Section, con una separación de $300 \mathrm{~m}$, dándole como referencia el modelo de elevación digital, asegurándose de que cada sección transversal sea lo suficientemente amplia como para cubrir la planicie de inundación.

Luego, utilizando la teoría de flujo inestable, se ingresó 18 valores de caudal promedio anual tomada desde el año 1997 hasta 2014, que fue calculado para la cuenca y corregido con el coeficiente de escorrentía de 0.45 ; estos datos van a ser insertados como hidrograma de flujo inestable en el software HEC-RAS. (Tabla 1).

El coeficiente de rugosidad (Coeficiente de Manning) se valorizó en diferentes tipos de espacios: el de corrientes naturales en orillas de 0.035 y para el fondo del canal 0.045 ; la pendiente aguas abajo fue de 0.0055 .

En relación con la pendiente de profundidad normal tanto en agua arriba, como aguas abajo, se va a tomar un alcance de 0.021875 para todos los perfiles; este resultado fue obtenido en base a las cotas máximo y mínimo del tramo principal.

Luego se obtuvo los datos para flujo estable; para calcular el caudal máximo en periodos de retorno de 30 , 50 y 100 años, usamos la distribución Gumbel (Tabla 2):

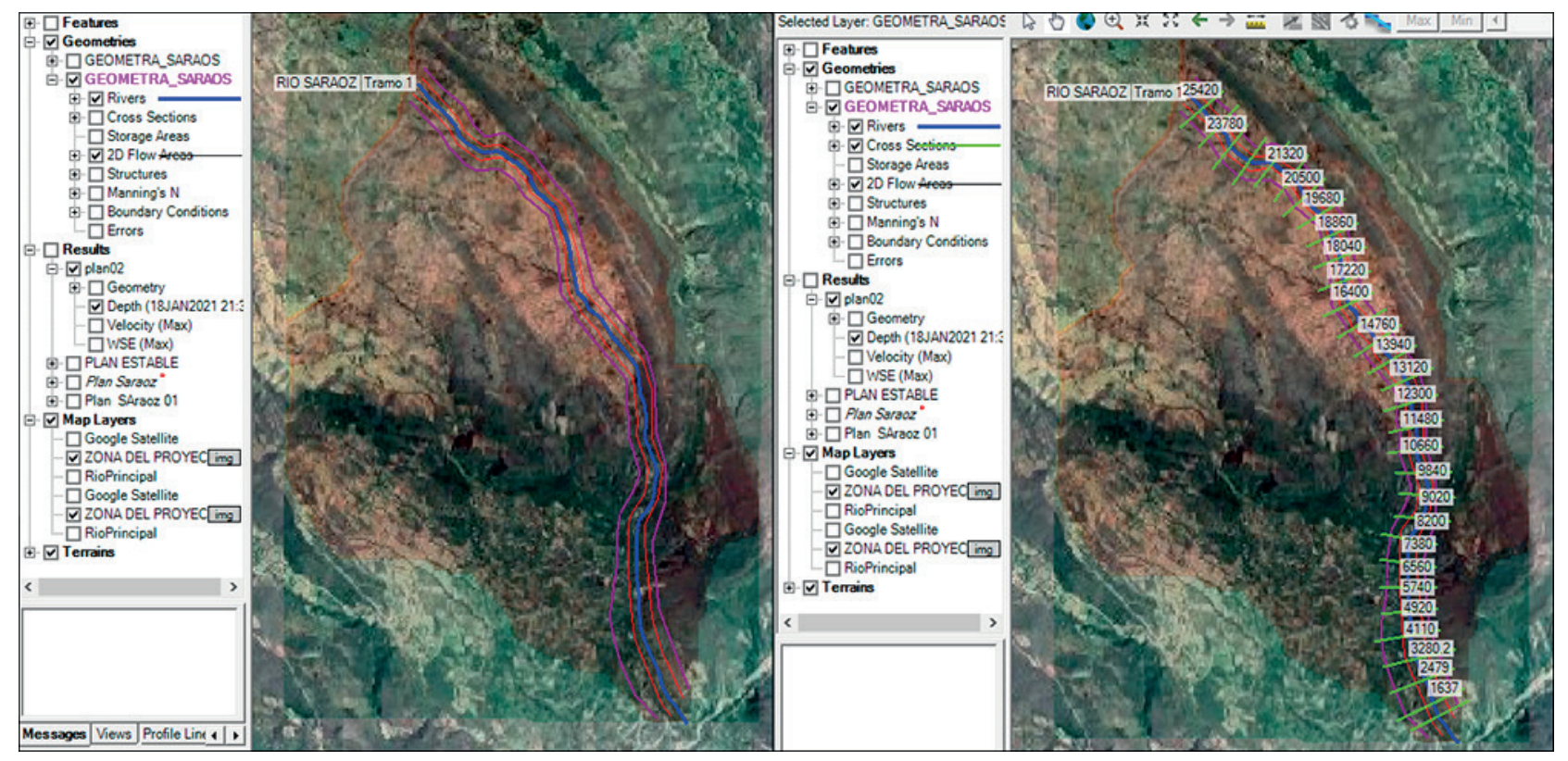

Figura 2. Determinación de los bancos de cauce y secciones, creadas cada 300 m 


$$
\mathrm{F}(\mathrm{y})=\mathrm{e}^{-\mathrm{e}^{\alpha(\mathrm{x}-\mu)}} \mathrm{Ec} .2
$$

Tabla 1. Caudales para el flujo inestable

\begin{tabular}{ccc}
\hline Número de años & Año & Caudales (m3/S) \\
\hline 1 & 1997 & 0.85 \\
2 & 1998 & 1.444 \\
3 & 1999 & 1.606 \\
4 & 2000 & 1.487 \\
5 & 2001 & 1.945 \\
6 & 2002 & 2.141 \\
7 & 2003 & 1.371 \\
8 & 2004 & 0.987 \\
9 & 2005 & 0.832 \\
10 & 2006 & 2.573 \\
11 & 2007 & 1.601 \\
12 & 2008 & 2.022 \\
13 & 2009 & 1.857 \\
14 & 2010 & 1.265 \\
15 & 2011 & 0.731 \\
16 & 2012 & 1.121 \\
17 & 2013 & 0.842 \\
18 & 2014 & 0.423 \\
\hline
\end{tabular}

Tabla 2. Caudales de diseño para 30, 50 y 100 años de retorno

\begin{tabular}{lllll}
\hline $\mathbf{N}^{\circ}$ & $\begin{array}{l}\text { Período de } \\
\text { retorno }\end{array}$ & Frecuencia & Probabilidad & $\begin{array}{l}\text { Distribución } \\
\text { Gumbel }\end{array}$ \\
\hline 1 & 30 & 0.033 & 0.967 & 2.951225 \\
2 & 50 & 0.020 & 0.980 & 3.232563 \\
3 & 100 & 0.010 & 0.990 & 3.612038 \\
\hline
\end{tabular}

\section{RESULTADOS Y DISCUSIONES}

Como proceso de simulación, utilizando el HEC-RAS, nos permitió identificar algunas zonas con mayor influencia de inundación (Figura 3). Dichas zonas corresponden a los inicios del curso de agua. Así como uno de los fenómenos que es importante resaltar es el cambio de curso del cauce principal por efecto de las corrientes de agua, el flujo turbulento y esporádicos flujos laminares con alta energía generan un proceso de erosión y transporte de materiales sedimentarios de diferentes diámetros, lo cual es depositado aleatoriamente, generando obstáculos del flujo de agua y cambiando direcciones de corriente como se observa en los transectos 20664, 9840, 8856, 984 entre otros (Figura 3).

En el proceso de inundación, se identifica tres zonas de mayor impacto (Figura 4); éstas crecidas de agua, alcanzan alturas de hasta $10 \mathrm{~m}$ de cota y anchos de hasta $140 \mathrm{~m}$. Esto nos muestra el potencial con la que se va generando la inundación con flujos turbulentos de remoción de masas y flujos laminares de mayor potencial que genera el desgaste de los materiales acumulados en los márgenes del cauce del río lo que genera un ensanchamiento del cauce de los ríos durante su recorrido en pendientes bajas de la Cordillera de los Andes. Un factor importante en el comportamiento de la erosión y el cambio de dirección del curso del río son las características topografías, los depósitos aluviales, así como la alta infiltración que ofrecen los tipos de materiales que son depositados a lo largo del cauce del río, lo que muestra una disminución de los flujos del caudal aguas abajo.

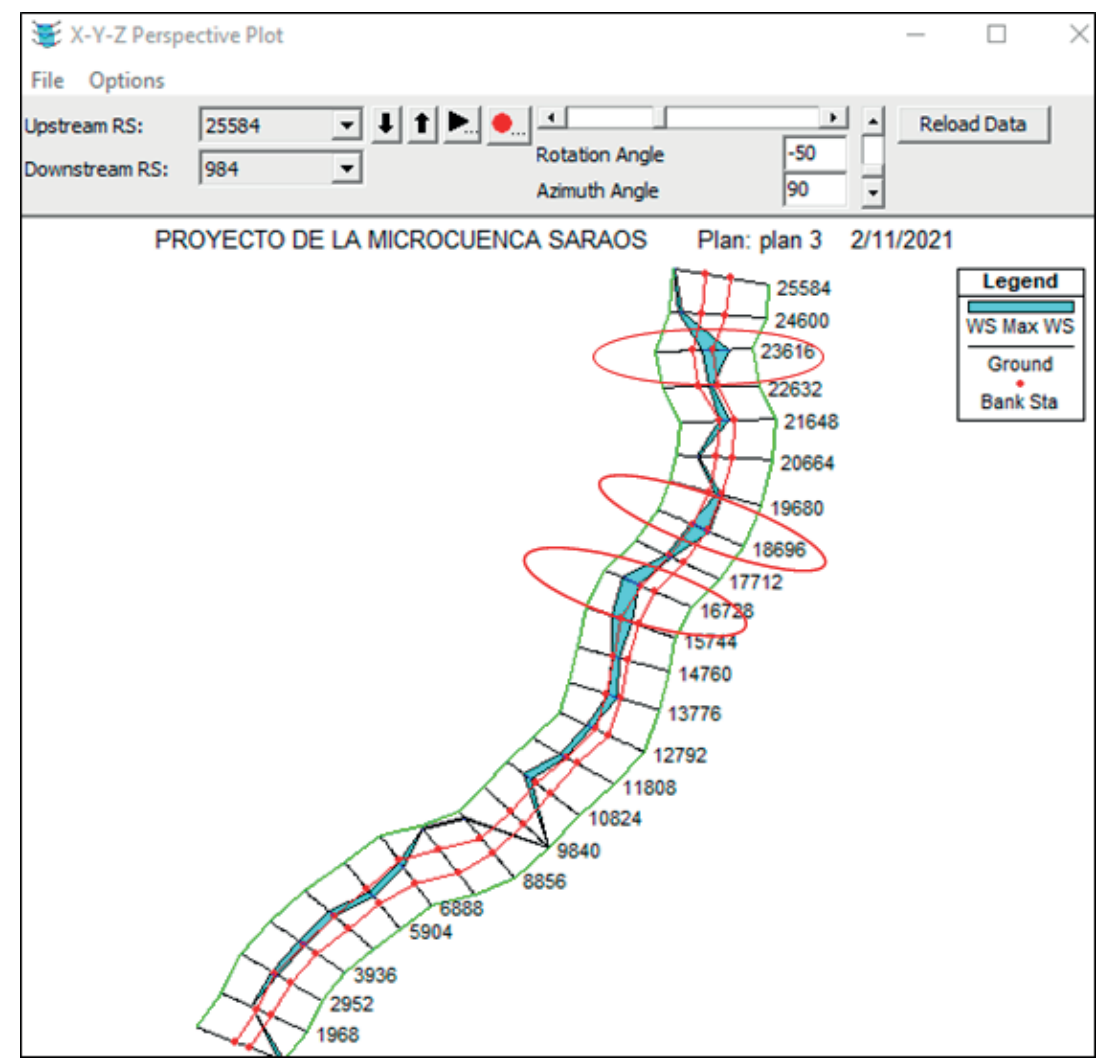

Figura 3. Flujo y manchas de inundación anual a lo largo de las secciones transversales del río Saraus. En círculos rojos se destaca las zonas de desbordes 
Asimismo, la Figura 5, muestra la simulación de inundación de las secciones transversales de la Figura 4 a través del Ras Mapper; donde la topografía es un factor influyente en la caracterización de los procesos de inundaciones, componente esencial de la geomorfología en cabeceras de cuenca (Alcántara Boza, 2019), la ilustración del lagunamiento de agua se genera en espacios con mayor abertura del cauce y desaparece en aquellos espacios encajonados, lo que trasciende nuevamente es la disminución del potencial de agua conforme va avanzando
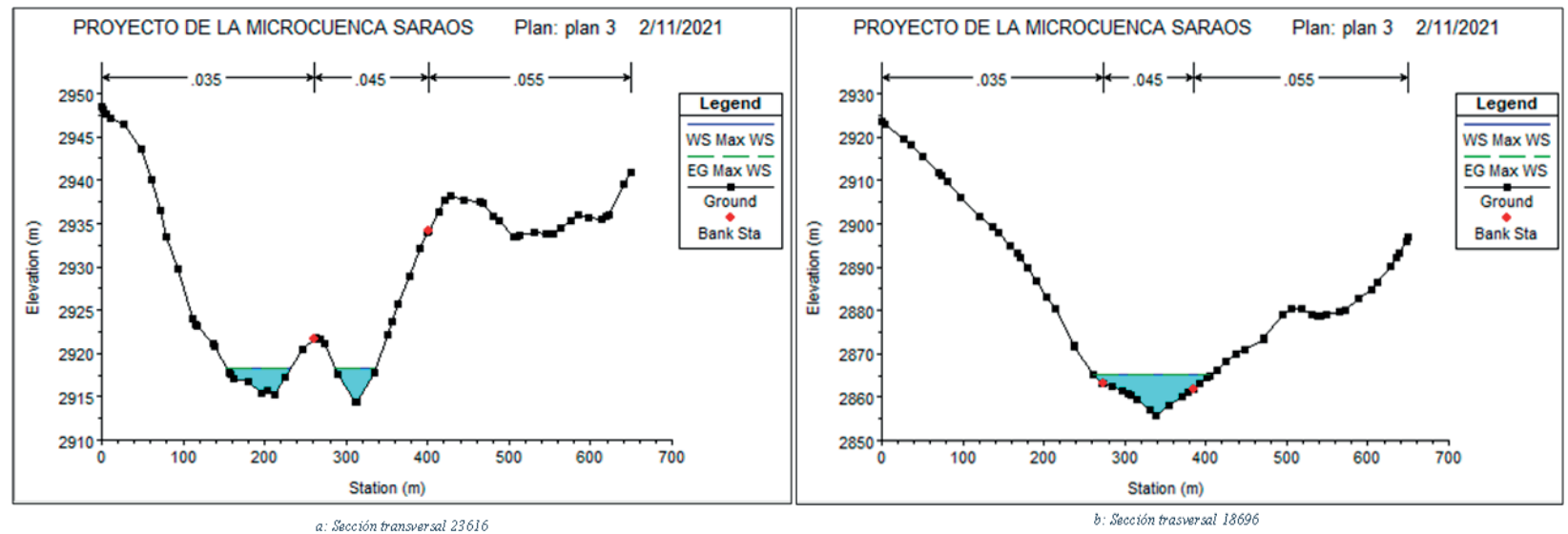

a: Sección transversal 23616

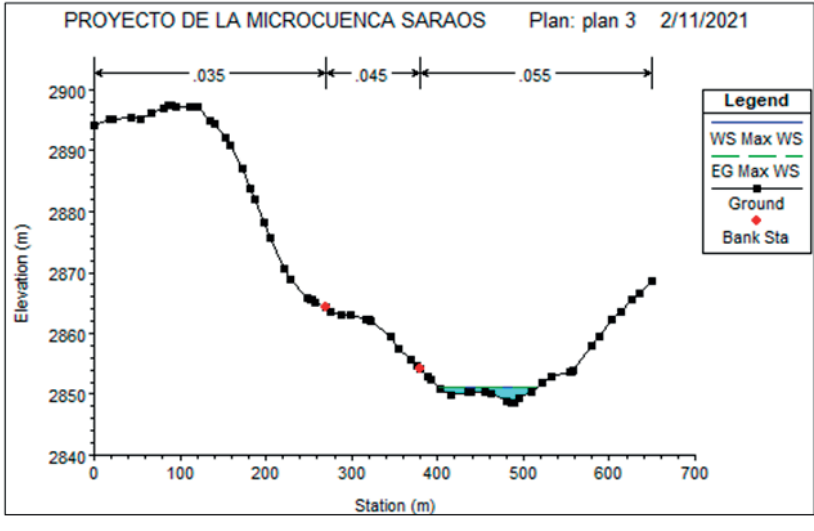

Figura 4. Sección trasversal 16728

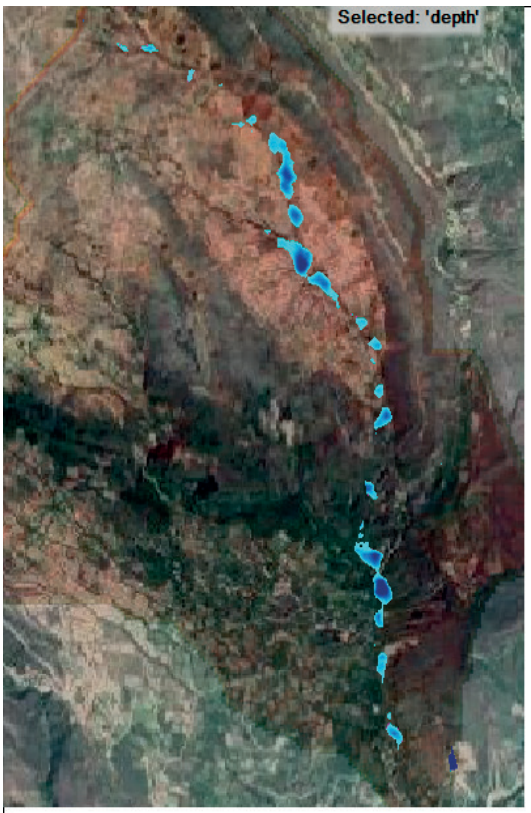

a) Inundación para $\mathbf{3 0}$ años

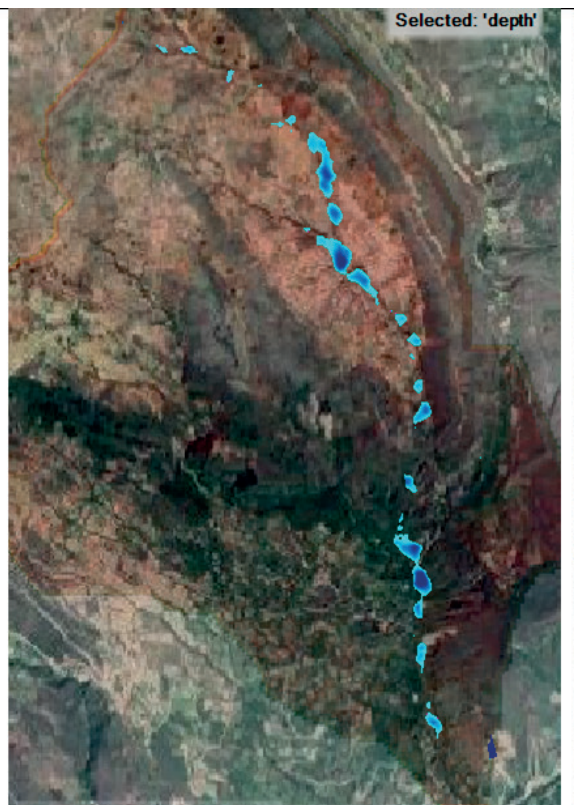

b) inundación para $\mathbf{5 0}$ años

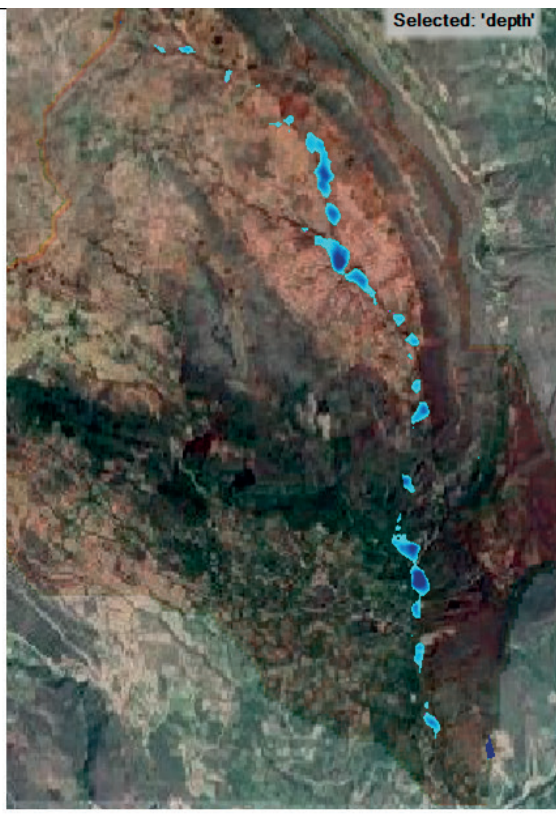

c) Inundación para 100 años

Figura 5. Simulación de inundación para tres periodos de retorno: 30, 50 y 100 años. 
las corrientes de agua y la distancia de recorrido de agua, ello estaría explicado por la fuerte infiltración durante el curso de agua.

En la Figura 5, se muestra el comportamiento de la simulación de flujo estable obtenidos para 3 periodos de retorno: 30, 50 y 100 años, donde se observa que no hay una marcada diferencia para las mismas zonas de inundaciones entre estos periodos de simulación, ello se encuentra asociado a la poca diferencia en los resultados de los caudales calculados para este tiempo de retorno, por lo que no existiría un cambio importante en la topografía a lo largo del tiempo; sin embargo no es uniforme el comportamiento de las inundaciones a lo largo de los transectos, como también lo mencionan Bilskie \& Hagen, (2018).

A diferencia de los ríos en la parte baja, el proceso de inundación es lento e incluso tardas horas, en cambio el proceso de inundación en la parte alta de las cordilleras es violento y de poca duración, como se observa en la figura 5, por ello el sistema de modelamiento es esencial en diversas áreas hidrológicas (I et al., 2019).

Con el fin de observar el efecto que tienen los tiempos de retorno de 30, 50 y 100 años, se muestra la figura 6, donde se observa la variación del caudal con un ligero incremento del nivel de cota. Para observar este cambio, se tomó como muestra la sección transversal 18696, donde se ha tomado en cuenta que la línea roja es referente al límite de cota o elevación del periodo de 30 años; así mismo, la línea morada para el periodo de retorno de 50 años y finalmente la línea azul, para el periodo de 100 años.

Una de las particularidades en la sierra y en aquellos espacios con bastante acumulación de material sedimentario es el uso para actividades agrícolas lo que es común en los grandes ríos (río Marañón, Ucayali, Ene,
Perené, entre otros), el mismo tipo de actividad pero en menor intensidad ocurre en los ríos de la parte media de la cordillera de los andes, lo cual serviría para implementar sistemas de alerta temprana para ríos de alta montaña (OEA, 2010); sin embargo, son estas las zonas más propensas a inundación donde el agricultor prácticamente lo va perdiendo todo, lo que no ocurre en algunos otros países donde se va generando un seguro contra inundaciones como lo mencionan Maccaferri et al, (2012), y en mucho de los espacios falta de un ordenamiento territorial con el uso no adecuado de zonas urbanas en cauces de río como lo explica Koslov (2019).

Es importante los datos que se han obtenido producto de esta simulación, en la cual el caudal de diseño para el periodo de retorno de 100 años es de $3.6 \mathrm{~m}^{3} / \mathrm{s}$, que, por las características de consistencia de los materiales acumulados en estos cauces de los ríos, implica la remoción de material detrítico en ciertas partes en el río principal de Saraus.

\section{CONCLUSIONES}

Los riesgos por inundación se identifican básicamente a inicios de la zona de estudio, ello debido a un alto incremento en los caudales de inicio, lo que conlleva a distintas zonas de inundación especialmente en zonas de poca pendiente, alcanzando alturas hasta $10 \mathrm{~m}$ de cota y anchos de $140 \mathrm{~m}$. esta dinámica de flujo también es el responsable de cambios de dirección y erosión de las riberas de los ríos.

Los periodos de retorno de 30, 50 y 100 años en estudio, no muestran una marcada diferencia en el incremento de la cantidad de caudal en la cuenca del río Saraus, por lo que los efectos también no muestran un importante modelamiento de las características geomorfológicas de la zona de estudio, por ser un río primario.

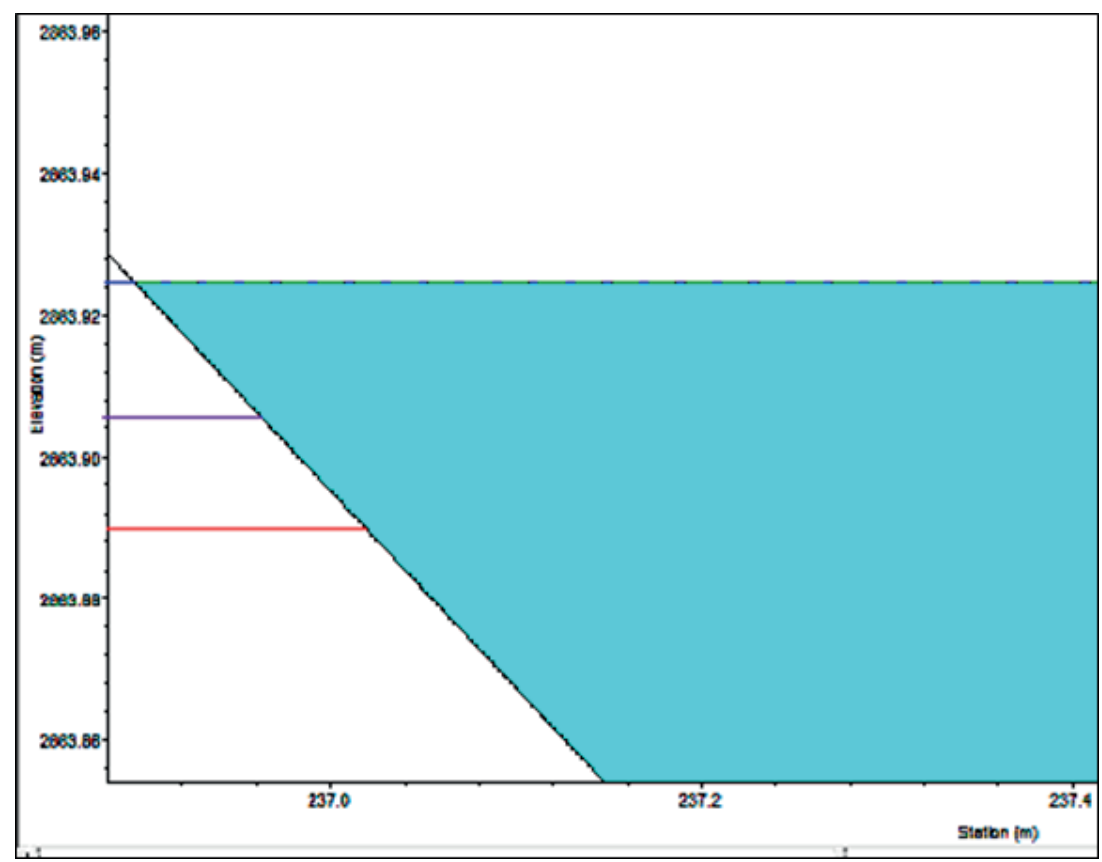

Figura 6. diferencia de cotas según el periodo de retorno 
Uno de los eventos poco revelados en este tipo de estudios es la influencia de la pendiente y el tipo de material sedimentario que se va acumulando en los cauces de este río, por lo que a ligeros incrementos del caudal, las posibilidades de erosión aumentan en función al tiempo y distancia, sin embargo, ello es disminuido por factores de infiltración que contrarresta el incremento de flujo a lo largo del cauce del río Saraus.

\section{REFERENCIAS}

Alcántara Boza, A. (2019). Influencia de la Geomorfología y la Meteorización en la Humedad del suelo y recargas de agua en la cabecera de cuenca del río Jequetepeque. In Revista del Instituto de Investigación de la Facultad de Ingeniería Geológica, Minera, Metalúrgica y Geográfica (Vol. 22, Issue 43). https://doi.org/10.15381/iigeo.v22i43.16694

Rodriguez, W \& Ortega, R. M. (2005). Aplicación SIG para evaluar el peligro de inundaciones. https://www.researchgate.net/ publication/313696640

Bilskie, M. V., \& Hagen, S. C. (2018). Defining Flood Zone Transitions in Low-Gradient Coastal Regions. Geophysical Research Letters, 45(6), 2761-2770. https://doi org/10.1002/2018GL077524

Brunner, G. (2010). HEC-RAS river analysis system, Hydraulic - USACE. US Army Corps of Engineers Hydrologic Engineering Center, Davis CA, January, 1-790. www.hec. usace.army.mil

Organización de los Estados Americanos. (2010). Manual para el diseño, instalación, operación mantenimiento de sistemas comunitarios de alerta temprana ante inundaciones. http:// www.rimd.org/advf/documentos/4ce3f9086d6db.pdf

Ceballos Lopez, J. D. (2011). Modelación hidráulica y morfodinámica de cauces sinuosos aplicación a la Quebrada La Marinilla (ANT). Boletín de Ciencias de La Tierra, 30, 107-118. https://revistas.unal.edu.co/index.php/rbct/article/ view/29299\#textoCompletoHTML

Fang, X., \& Pomeroy, J. W. (2016). Impact of antecedent conditions on simulations of a flood in a mountain headwater basin. Hydrological Processes, 30(16), 2754-2772. https:// doi.org/10.1002/hyp.10910

I, F. V., C, C. C., B, F. A., \& V, L. L. (2019). Pronóstico de precipitación sobre la cuenca aportante al embalse " La Esperanza " aplicando modelo climático HadCM3 Precipitation forecast on the basin contributing to the " La Esperanza " reservoir using HadCM3 climate model. Revista Del Instituto de Investigación de La Facultad de Minas, Metalurgia y Ciencias Geográficas, 22, 29-36. https://revistasinvestigacion.unmsm.edu.pe/index.php/ iigeo/article/view/16683

Koslov, L. (2019). How maps make time: Temporal conflicts of life in the flood zone. City, 23(4-5), 658-672. https://doi.or $\mathrm{g} / 10.1080 / 13604813.2019 .1690337$

Maccaferri, S., Cariboni, F. and Campolongo, F. (2012). Natural Catastrophes: Risk relevance and insurance coverage in the EU. https://ec.europa.eu/info/sites/default/files/jrc-reporton-natural-catastrophes_en.pdf
Mohamed, A.M., Abdelllah, A., Ahmed, A., Kamal, E., Zouhair, O. and Abdelouhed, F. (2020). Flood disaster simulation and predicting using her-ras and gis tools: A case of the rdat river's downstream. Disaster Advances Journal. volume 13, Issue 11, pagees 10-17. https://www.scopus.com/record/ display.uri?eid=2-s2.0-85094151731\&origin=resultslist

Nanía, L. S., \& Molero, E. (2007). Manual básico de HEC-RAS 3.1.3 y HEC- GeoRas 3.1.1. Universidad de Granada. Area de Ingeniería Hidráulica, 1-59. https://cemexico.groups. et.byu.net/vocabulary/ManualBasico_HEC-RAS313 HECGeoRAS311_Espanol.pdf

Radzicki, K,, Goleviowski, T., Cwilkid, M. and Stolinski. M. (2021). A new levee control system based on geotechnical an geophysical surveys indluding active thermal sensing: A case study fron polando. Engineering Geology Journal. Volume 239, November 2021 106316. https://doi. org/10.1016/j.enggeo.2021.106316

Quast, J., Böhme, M., Ehlert, V., Ette, J., Gottschick, M., Jaeckel, A., Knierim, A., Messal, H., Sawicka, M., Sbjeschni, A., Schmidt, W., Szerencsits, M., \& von Tümpling, W. (2011). Flood risks in consequence of agrarian land-use measures in flood formation and inundation zones and conclusions for flood risk management plans. Irrigation and Drainage, 60(SUPPL. 1), 105-112. https://doi.org/10.1002/ird.674

Quisphe Torres, Forge Armando; Sullca Otazu, R. F. (2015). Aplicación del Modelo Matemático HEC RAS Para el Cálculo del Perfil Hidráulico del Río Ramis [Universidad Andina Nestor Caceres Velasquez]. http://repositorio.uancv. edu.pe/handle/UANCV/82

Shivaprasad Sharma SV, Parth Sarathi Roy, Chakravarthi V, S. G. \& B. V. (2017). Extraction of detailed level flood hazard zones using multi-temporal historical satellite data-sets - a case study of Kopili River Basin, Assam, India. Geomatics, Natural Hazards and Risk, 792-802. https://www. tandfonline.com/doi/full/10.1080/19475705.2016.1265014

Solín, Madajová, M. S., \& Michaleje, L. (2017). Flood hazards in the headwaters area: Lessons learned from a survey of households in the upper Myjava basin, Slovakia. Water Policy, 19(6), 1081-1096. https://doi.org/10.2166/ wp.2017.008

Talha Anees, M., Abdullah, K., Nawawi, M. N. M., Nik Norulaini, N. N. N., Ismail, A. Z., Syakir, M. I., \& Abdul Kadir, M. O. (2019). Prioritization of Flood Vulnerability Zones Using Remote Sensing and GIS for Hydrological Modelling. Irrigation and Drainage, 68(2), 176-190. https://doi. org/10.1002/ird.2293

Ardiclioglu, M. And Kuriqi, A. (2019). Calibration of chanel roughness in intermittent rivers using HEC-RAS model: case of Sarimsakli creek, Turkey. SN APPLIED SCIENCES. Volume 1. Issue: 9 . https://doi.org/10.1007/s42452-019-1141-9

Turégano, F. (2012). Rio Valdemembra a su paso por Motilla del Palancar - Gestión de riesgo de inundación [UPM. España]. http://oa.upm.es/14935/

Wang, L. ling, Yao, W. yi, Tang, J. liang, Wang, W. long, \& Hou, X. xin. (2018). Identifying the driving factors of sediment delivery ratio on individual flood events in a long-term monitoring headwater basin. Journal of Mountain Science, 15(8), 18251835. https://doi.org/10.1007/s11629-017-4739-7 